\title{
Análisis de las propiedades psicométricas de la Escala de Autoconcepto de Piers-Harris 2 en escolares mexicanos
}

\section{Analysis of the psychometric properties of the Piers-Harris Self-Concept Scale 2 in Mexican school children}

\author{
López-García, Danivia ; Hernández-Padilla, Eduardo ${ }^{1}$ y Palacios-Hernández, Bruma ${ }^{1}$
}

\begin{abstract}
Resumen:
Actualmente, en México existe escasez de instrumentos válidos para evaluar el autoconcepto en niños, por ello el objetivo del presente trabajo fue analizar las propiedades psicométricas de la adaptación de la Escala de Autoconcepto de Piers-Harris 2 en 493 escolares de 7 a 12 años (mujeres 50.5\%), con un promedio de nivel escolar de cuarto grado. El estudio fue transversal cuantitativo con enfoque empírico analítico no experimental. Se evaluó la consistencia interna empleando la fórmula KR20, obteniendo resultados semejantes a otros estudios (índice $=0.87$ ). El análisis con modelamiento Rasch, indica que 58 reactivos de la escala obtuvieron buenas propiedades de validez, confiabilidad y ajuste. Dicha escala presenta propiedades psicométricas adecuadas para ser utilizada en niños escolarizados de 7 a 12 años de población mexicana.
\end{abstract}

\begin{abstract}
:
Currently, in Mexico there are few valid instruments to evaluate self-concept in children, so the objective of this work was to analyze the psychometric properties of the adaptation of the PiersHarris Self-Concept Scale 2 in 493 school children from 7 to 12 years of age (female $50.5 \%$ ), with an average fourth grade school level. The study was transversal quantitative with a non-experimental analytical empirical approach. Internal consistency was evaluated using the KR20 formula, with results similar to other studies (index. 0.87). Rasch modeled analysis indicates that 58 reagents on the scale obtained good validity, reliability, and fit properties. This scale has adequate psychometric properties for its use in Mexican schoolchildren from 7 to 12 years old.
\end{abstract}

Keywords: Scale Piers Harris 2, Self-concept, Rasch Modeling, Schoolchildren, Mexico.
Palabras Clave: Escala de autoconcepto Piers Harris 2, Autoconcepto, Modelamiento Rasch, Escolares, México.

\footnotetext{
${ }^{1}$ Centro de Investigación Transdisciplinar en Psicología. Universidad Autónoma del Estado de Morelos. México
}

*Correspondencia: danivia3@hotmail.com 
Durante las últimas décadas se ha subrayado desde diferentes ámbitos de la psicología, la importancia del autoconcepto en el bienestar subjetivo como un concepto central para el estudio y comprensión del comportamiento humano y su interacción con el entorno. $\mathrm{Su}$ efecto es bidireccional, ya que diversos estudios han coincidido en que el autoconcepto va a tener una función mediadora en el sujeto que le permitirá o impedirá realizar una tarea como resultado de la valoración de sí mismo, brindando una doble función: la de condicionar y retroalimentar el propio desempeño (Gorostegui, 2010). Esta función mediadora del autoconcepto se ha comprobado relevante en población de niños en la educación primaria (Galindo-Domínguez, 2019; Guay, Marsh \& Boivin, 2003).

Cazalla \& Molero (2013) definieron al autoconcepto como un constructo jerárquico y con múltiples dimensiones resultado del proceso en donde la persona es capaz de construir una conciencia de sí misma a partir de las percepciones y experiencias derivadas de su interacción y experiencia desarrollada con los demás. Si bien el estudio del autoconcepto ha sido abordado por diferentes perspectivas teóricas, desde un aspecto global, Shavelson, Hubner y Stanton (1976), definen que el término autoconcepto se refiere a la autopercepción de las personas con relación a aspectos o dimensiones importantes de sus vidas. Estas percepciones están influidas por factores biológicos y culturales, y se van desarrollando principalmente mediante la interacción del individuo con el medio ambiente durante la niñez, y por actitudes y comportamientos de otros, dando lugar a actitudes y sentimientos autoevaluativos que tienen importantes funciones organizativas y motivan el comportamiento. Con el tiempo, un autoconcepto individual puede cambiar en res- puesta al medio ambiente, como la familia, el círculo social y el ambiente escolar o desarrollar cambios mentales como resultado de cambios en las prioridades o valores, aunque, estos cambios en general no ocurren rápidamente o en respuesta a experiencias o intervenciones aisladas (Piers \& Herzberg, 2002).

El estudio del autoconcepto y su relación con el desempeño académico en niños de educación primaria ha sido extenso y ha reportado una asociación entre niveles positivos de autoconcepto, en especial el autoconcepto académico/intelectual $\mathrm{y}$ altos niveles de desempeño escolar (Cvencek, Fryberg, Covarrubias, \& Metzoff, 2018; Fives, 2016; Susperreguy, Davis-Kean, Duckworth \& Chen, 2018,). Se han mostrado en especial, más bajos niveles de autoconcepto en poblaciones de escolares con dificultades diversas de salud, como niños con Trastornos de atención e hiperactividad (Dolgun, Savaşer \& Yazgan, 2014), epilepsia (Ekinci et al., 2016) espina bífida (Shields, Taylor \& Dodd, 2008), ideación suicida (Chávez-Hernández et al., 2018) y labio y/o paladar hendido (Ha, Shi \& Zheng, 2015) en comparación a escolares sin afectaciones relevantes de salud. Los resultados de los diversos estudios reportan una interrelación significativa entre el autoconcepto de los niños, su desempeño académico y la salud de los mismos que aportan indicadores para diseñar estrategias educativas, familiares y de salud específicas para promover un mejor desempeño en las esferas escolares e interpersonales.

Existe un limitado número de instrumentos desarrollados o validados en México que evalúan el autoconcepto de manera global o multidimensional en niños mexicanos, y su mayoría han sido dirigidos principalmente a estudiar población adolescente, como la Escala Tetradimensional de Autoconcepto para 
Adolescentes (ETAA) desarrollado por Guido, Mújica y Gutiérrez (2011), la Escala de Autoconcepto Adolescentes y Adulto (EAAA) por Pineda y Pérez (2015) y la adaptación del Autoconcepto Forma 5 (AF5) diseñado por García y Musitu (1999), realizada por Salum-Fares, Marín y Reyes (2011). Uno de los instrumentos de evaluación del autoconcepto en población infantil más usado, es la Escala de Autoconcepto de Piers-Harris en su segunda edición (Piers, 1984), desarrollada originalmente a principios de la década de 1960 para proporcionar un breve instrumento de autoinforme para la evaluación del autoconcepto en niños y adolescentes.

Desde su introducción, la escala ha disfrutado de una amplia aceptación entre profesionales e investigadores (Alexopoulos \& Foudoulaki, 2002; Dolgun, Savaşer \& Yazgan, 2014; Flahive, Chuang \& Li, 2011; Flahive, Chuang \& Li, 2015; Goltz \& Brown, 2014, Gorostegui, 1992). El desarrollo de esta escala se basó en la opinión de los individuos acerca de sus creencias relativamente coherentes sobre sí mismos, creencias que se desarrollan y se estabilizan durante la niñez. Este conjunto de creencias representa un concepto propio de las personas, un término que algunos investigadores han utilizado indistintamente con conceptos como la autoestima y el autorespeto. Piers y Herzberg (2002), asumieron que los niños revelarían aspectos importantes de su autoimagen subyacente consensuando o discrepando de declaraciones simples, autodescriptivas, y que esta evaluación de su representación sobre sí mismo se relacionaría significativamente con otros aspectos de la personalidad y con las predicciones del comportamiento futuro. El Piers-Harris 2 incorporó datos normativos representativos basados en una muestra de 1.387 jóvenes, de 7 a 18 años, reclutados de distritos escolares de todo Estados Unidos y redujo la escala de 80 a 60 ítems. Esta reducción acorta significativamente el tiempo de administración, y a su vez conserva todas las escalas de autoconcepto y validez de la escala original (Piers \& Herzberg, 2002).

La necesidad de realizar adaptaciones socioculturales en instrumentos de evaluación del autoconcepto es altamente relevante dada la influencia significativa de las características del entorno y del tipo de interacciones y experiencias a las que se ve expuesto el sujeto y afectan en la construcción de la autovaloración de sí mismo (Heine, 2001) con posibilidad de alteración de la confiabilidad y validez de los instrumentos dependiendo el contexto sociocultural en el que se evalúa (Alrajhi et al., 2019; Flahive, Chuang \& Li, 2015).

Debido a la escasez de instrumentos válidos y confiables para evaluar el autoconcepto en población infantil mexicana, se diseñó e implementó un estudio, con el objetivo de adaptar y analizar las propiedades psicométricas de la Escala de Autoconcepto de Piers-Harris 2 en una muestra de niños escolarizados mexicanos de 7 a 12 años.

\section{Método}

El presente estudio contó con un diseño transversal cuantitativo con enfoque empírico analítico no experimental.

\section{Participantes}

La muestra participante estuvo conformada por 493 sujetos $(50.5 \%$ hombres, $49.5 \%$ mujeres), provenientes de tres municipios del estado de Morelos, México; con un nivel escolar en sistema público de segundo a sexto año de primaria y un rango de edad de 7 a 12 años. Los criterios de inclusión incluyeron que se tratara de niños, hispanohablantes nativos, hijos de padres que hablan el español, sin diagnóstico de alguna alteración cognoscitiva 
y/o socioafectiva que pudieran influir sobre los resultados.

\section{Instrumentos}

La escala de autoconcepto Piers-Harris 2 (Piers, Harris \& Herzberg, 2002), evalúa la percepción que un niño de 7 a 12 años tiene de sí mismo respecto de sus actitudes, sus sentimientos y el conocimiento de sus capacidades, habilidades, apariencia, y aceptabilidad social. Esta escala consta de 60 frases sencillas con respuesta dicotómica (SI-NO) en las que se pide al niño/niña que decida por alguna de las dos opciones. La puntuación se otorga en una escala de 5 a 99, según el baremo de cada nivel que indica el porcentaje de sujetos que se encuentran por debajo de la puntuación directa correspondiente. Los autores han sugerido que un centil 39 o menor indicaría que el niño tiene un autoconcepto bajo, $\mathrm{y}$, un centil de 60 o más representa un autoconcepto alto. En su validación original, se obtuvo un coeficiente Alfa de Conbrach global de 0,91 y en las diferentes subescalas de 0.81 (BEH, Comportamiento de ajuste), 0,81 (INT, estado intelectual), 0.75 (PHY, Aspecto físico y atributos), 0.81 (FRE, libre de ansiedad), 0.74 (POP, popularidad) y 0.77 (HAP, felicidad y satisfacción).

La escala permite obtener información sobre la percepción global que niño tiene de sí mismo, y cómo valora seis aspectos de su forma de ser y su comportamiento, según las siguientes 6 dimensiones:

- Comportamiento de ajuste (BEH): reporta medidas de admisión o el rechazo de comportamientos problemáticos (reactivos 12, 13, 14, 18, 19, 20, 27, 30, $36,38,45,48,58$ у 60).

- $\quad$ Estado intelectual (INT): refleja la evaluación de un niño de sus habilidades con respecto a las tareas intelectuales y académicas (reactivos $5,7,12,16,18$, $21,22,24,25,26,34,39,43,50,52$ у 55).

- $\quad$ Aspecto físico y atributos (PHY): medidas de valoración de un joven de su apariencia física y atributos como el liderazgo y la habilidad de expresar ideas (reactivos 5, 8, 9, 15, 26, 33, 39, 44, 46, 49 y 54).

- $\quad$ Libre de ansiedad (FRE): el puntaje refleja ansiedad y estado de ánimo disfórico $(4,6,7,8,10,17,23,29,31,32$, $35,40,56$ y 59$)$

- $\quad$ Popularidad (POP): representa la evaluación de un niño sobre su funcionamiento social (reactivos 1, 3, 6, 11, 32, 37, 39, 41, 47, 51, 54, 57).

- $\quad$ Felicidad y satisfacción (HAP): refleja el sentimiento de felicidad y satisfacción con la vida (reactivos 2, 8, 28, 31, $35,40,42,49,53,60)$.

\section{Procedimiento}

Traducción y Adaptación de la Escala.

Se llevó a cabo una traducción independiente del inglés al español, cuya versión se comparó con la versión en español realizada por Alejandro Sánchez, M.A. (Copyright (C) 2002 by Ellen V. Piers and Dale B. Harris. Publicado por W.P.S. Todos los derechos reservados). La versión en español se sometió también a revisión de tres expertos profesionales en diversas áreas de la Psicología y dedicados a la investigación con práctica en la aplicación y validación de instrumentos de medición, hispanoparlantes nativos mexicanos, lo cual permitió corroborar que la traducción haya sido la adecuada.

De acuerdo con las observaciones realizadas por los expertos, se realizaron adecuaciones en la redacción de los ítems: 3, 4, 11, $13,17,18,24,32,33,40,42,43,44,46,47$, 
48, 54 y 55, para mantener el orden y el sentido original de la escala, con la cual se obtuvo una versión final. Esta versión fue analizada por un grupo de maestros de nivel primaria, con la finalidad de comprobar que los niños pudieran entender las consignas y comprensión de los ítems, así como su adaptación sociocultural de los términos usados. Esta revisión validó la redacción del instrumento, considerando que el lenguaje era simple, adecuado y adaptado al contexto mexicano para la comprensión de los alumnos de nivel primaria.

\section{Procesamiento y análisis de datos}

La información recopilada en la versión impresa de la escala fue digitada, binariamente en el software Excel(C), posteriormente se corrigieron los errores detectados; y, las bases de datos fueron transferidas a los softwares IBM-SPSS 23.0C, Winstep 3.81C, y, Rasch (. Para llevar a cabo los análisis factoriales confirmatorios se usó el software $\mathrm{R} v$. 3.5.1 (R Development Core Team, 2018).

Los aspectos evaluados de la escala general y de sus dimensiones fueron: I) los valores de consistencia interna de la escala general y de sus dimensiones, comparando los resultados con algunos criterios o estándares sugeridos (George \& Mallery, 2003; Streiner, 2003); II) el ajuste de los datos al modelo utilizando el criterio de ajuste Infit, que detecta desajustes en respuestas cerca de la zona de medición del ítem; y, el Outfit, sensible a los desajustes lejos de la zona de medición del ítem, es decir, puede ser afectado por los casos atípicos, donde el criterio de aceptación para ambos se ubica entre el 0.5 y el 1.5 lógitos; III) comparación de la probabilidad de respuesta de los ítems de la escala, con la capacidad de los participantes para responder el instrumento utilizando el mapa de Wright $($; IV) finalmente, se realizó un análisis factorial exploratorio mediante el empleo de los com- ponentes principales y el tipo de rotación Promax.

Análisis de consistencia interna

Se obtuvieron los índices de confiabilidad Kuder-Richardson 20 y el coeficiente Alfa de Cronbach; el primero de ellos, debido a que las opciones de respuesta son dicotómicas, y correspondería la fiabilidad KR20 (Kuder \& Richardson, 1937; Zimmerman, 1972; Becker, 2008); adicionalmente distintos trabajos (Flahive, Chuang \& Li, 2015; Piers \& Herzberg, 2002) han empleado el coeficiente Alfa de Cronbach.

Estrategia de análisis factorial confirmatorio Pérez-Gil, Chacón y Moreno (2000) mencionan que, entre los métodos estadísticos más populares para la validación de constructo de una prueba, esto es, para evidenciar empíricamente que el test corresponde al constructo que pretende medir, son el Análisis Factorial Exploratorio (AFE) y el Análisis Factorial Confirmatorio (AFC). El AFC busca comprobar el ajuste de los datos a un modelo teórico previamente establecido, y de la misma manera evidenciar si los reactivos se agrupan según las dimensiones que pretendidamente subyacen a su elaboración. Diversos estudios reportan distintas soluciones al análisis factorial, y se ha identificado inconsistencia en el número de factores y reactivos que conforman el Piers-Harris 2 en estudios que lo han adaptado en distintos países (Butler \& Gasson, 2005; Lewis \& Knight, 2000; Collins; Kafer \& Shea, 1985), lo cual podría sugerir la ausencia de una teoría consolidada sobre el constructo.

\section{Consideraciones éticas}

El estudio fue autorizado por las instituciones escolares en donde se reclutó la muestra y contó con el consentimiento informado por parte de los padres y/o tutores, así como el 
asentimiento por parte de los niños que participaron en el estudio, a quienes se respondió a toda pregunta que formularon a lo largo del estudio.

\section{Resultados}

En la Figura 1 se muestra el modelo de medición propuesto para el Análisis Factorial Confirmatorio; en donde los diferentes reactivos son representados por rectángulos en tanto que las dimensiones lo son por un óvalo. Las relaciones de asociación de reactivos a las dimensiones se indican mediante las líneas que los unen; cabe señalar que existen reactivos que representan más de una dimensión. El análisis factorial confirmatorio reconoció la estructura propuesta de construcción de la Escala de autoconcepto de Piers-Harris 2, sus dimensiones y sus reactivos (Piers, \& Herzberg, 2002). Se observó la naturaleza dicotómica de los reactivos con la finalidad de obtener los resultados indicados acerca de la estructura del constructo.

El análisis de consistencia interna tanto para el alfa de Cronbach como la prueba $\mathrm{Ku}$ der-Richardson 20 son mostrados en la Tabla 1 , donde puede apreciarse que los índices obtenidos por ambos análisis son prácticamente iguales en la escala general y en las dimensiones que las conforman, con coeficientes que se oscilan entre el rango cuestionable y bueno. Solamente la dimensión de Popularidad obtuvo un coeficiente insatisfactorio (0.57) (George \& Mallery, 2003; Streiner, 2003).

Tabla 1.

Comparación de valores de consistencia interna de los índices de consistencia interna alfa de Cronbach y Kuder-Richardson 20 para la escala general de Autoconcepto de Piers-Harris, y las seis dimensiones que la conforman.

\begin{tabular}{lcc}
\hline Dimensión & $\begin{array}{c}\text { Alfa de } \\
\text { Cronbach }\end{array}$ & $\begin{array}{c}\text { Kuder Richard- } \\
\text { son 20 }\end{array}$ \\
\hline Total & 0.87 & 0.87 \\
Comportamiento & 0.76 & 0.76 \\
Intelectual & 0.72 & 0.71 \\
Físico & 0.69 & 0.69 \\
Ansiedad & 0.70 & 0.70 \\
Popularidad & 0.57 & 0.57 \\
Satisfacción & 0.66 & 0.66 \\
\hline
\end{tabular}

Nota: Elaboración propia con base a los análisis de fiabilidad realizados a la prueba y sus dimensiones. 


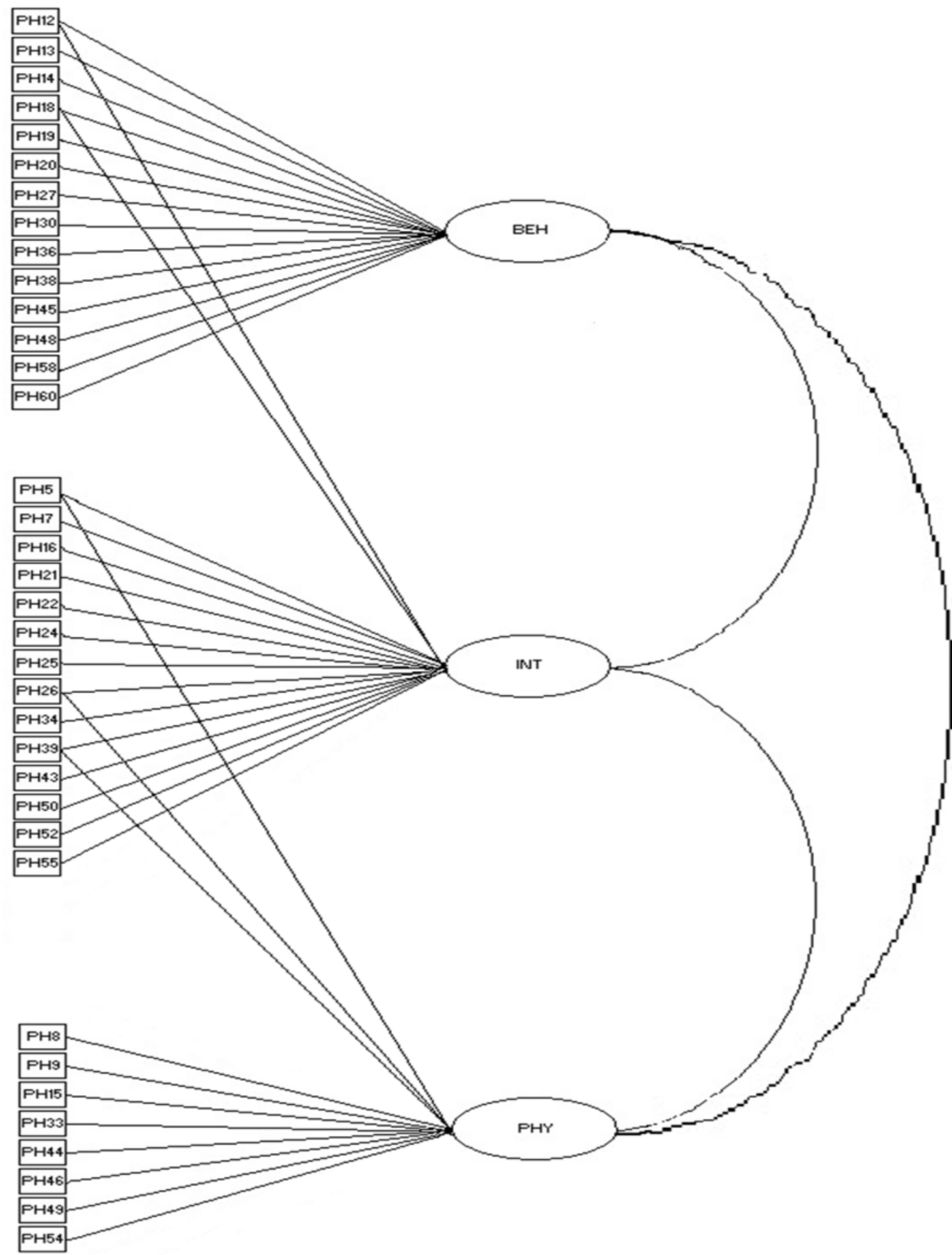

Figura 1. Representación gráfica del modelo de medición (AFC) de las dimensiones BEH, INT, PHY y sus correspondientes reactivos de la Escala de Autoconcepto Piers-Harris 2 (por cuestiones de espacio y simplicidad solamente se emplean tres dimensiones). Fuente: Elaboración propia con base a la estructura de la Escala de Autoconcepto de Pier-Harris y las dimensiones que la constituyen. 
Los resultados del análisis factorial confirmatorio mostrados en la Tabla 2, indican el valor de los coeficientes de los reactivos por dimensión a la que corresponden de la escala de Autoconcepto Piers-Harris. En dicha tabla pueden observarse que la gran mayoría de los coeficientes de los reactivos tienen un valor positivo y son estadísticamente significativos en las dimensiones BEH e INT a las que corresponden. Las magnitudes de dichos coeficientes en su gran mayoría asumieron valores por encima de 0.20 el monto del incremento, en contraste sólo tres reactivos reportaron una baja importancia en la representación de la dimensión. Para los coeficientes obtenidos en las dimensiones PHY, FRE, HAP y, POP, aunque algunos de ellos resultaron positivos y estadísticamente significativos, por su parte otros asumieron valores negativos con significancia formal, y también resultaron en su mayoría positivos y estadísticamente significativos. Debe señalarse que en estas dimensiones se encuentra un número significativo de coeficientes que no tienen una relación estadística con su dimensión.

Tabla 2.

Resultados del análisis factorial confirmatorio AFC en la versión Piers-Harris 2 para las dimensiones, en la muestra de niños mexicanos. Solución completamente estandarizada.

\begin{tabular}{|c|c|c|c|c|c|c|c|c|c|c|c|}
\hline \multicolumn{4}{|c|}{$\begin{array}{l}\text { Comportamiento de ajuste } \\
\text { (BEH) }\end{array}$} & \multicolumn{4}{|c|}{ Estado intelectual (INT) } & \multicolumn{4}{|c|}{ Aspecto físico y atributos (PHY) } \\
\hline PH12 & 0.42 & - & - & PH5 & 0.43 & - & - & PH5 & 0.15 & - & - \\
\hline $\mathrm{PH} 13$ & 0.44 & -0.25 & 0 & PH7 7 & 0.18 & -0.2 & 0.02 & PH8 & -0.17 & -0.47 & 0.05 \\
\hline $\mathrm{PH} 14$ & 0.49 & -0.24 & 0 & PH12 & 0.05 & -0.13 & 0.21 & PH9 & 0.38 & -1.19 & 0.01 \\
\hline $\mathrm{PH} 18$ & 0.14 & -0.18 & 0.06 & PH16 & 0 & -0.13 & 0.89 & PH15 & 0.27 & -0.79 & 0.01 \\
\hline PH19 & 0.67 & -0.27 & 0 & PH1 8 & 0.35 & -0.16 & 0 & $\mathrm{PH} 26$ & 0.23 & -0.71 & 0.02 \\
\hline PH2O & 0.37 & -0.24 & 0 & $\mathrm{PH} 21$ & 0.54 & -0.22 & 0 & PH33 & 0.48 & -1.29 & 0.01 \\
\hline PH27 & 0.72 & -0.41 & 0 & $\mathrm{PH} 22$ & 0.13 & -0.17 & 0.47 & PH39 & 0.28 & -0.9 & 0.01 \\
\hline PH30 & 0.33 & -0.48 & 0.02 & $\mathrm{PH}_{24}$ & 0.36 & -0.18 & 0 & PH44 & 0.53 & -1.55 & 0.01 \\
\hline PH36 & 0.47 & -0.2 & 0 & $\mathrm{PH} 25$ & 0.49 & -0.29 & 0 & PH46 & 0.93 & -3.08 & 0.03 \\
\hline PH38 & 0.57 & -0.29 & 0 & $\mathrm{PH} 26$ & 0.32 & -0.17 & 0 & PH49 & 0.22 & -0.69 & 0.03 \\
\hline PH45 & 0.55 & -0.28 & 0 & $\mathrm{PH} 34$ & 0.24 & -0.29 & 0.01 & РH54 & 0.55 & -1.67 & 0.01 \\
\hline PH48 & 0.38 & -0.18 & 0 & PH39 & 0.27 & -0.2 & 0 & $\mathrm{PH} 30$ & 0.11 & -0.94 & 0.24 \\
\hline PH58 & 0.43 & -0.24 & 0 & PH43 & 0.21 & -0.15 & 0.01 & PH16 & 0.18 & -0.55 & 0.06 \\
\hline PH60 & 0.23 & -0.14 & 0 & PH5O & -0.1 & -0.12 & 0.16 & $\mathrm{PH}_{2} 2$ & 0.31 & -1.06 & 0.01 \\
\hline $\mathrm{PH} 25$ & 0.3 & -0.32 & 0 & PH52 & 0.49 & -0.22 & 0 & PH34 & 0.24 & -0.88 & 0.02 \\
\hline $\mathrm{PH} 34$ & -0.15 & -0.31 & 0.06 & PH55 & 0.15 & -0.18 & 0.03 & $\mathrm{PH} 50$ & 0.09 & -0.38 & 0.22 \\
\hline PH55 & 0.26 & -0.24 & 0 & РH30 & -0.16 & -0.3 & 0.09 & PH41 & 0.2 & -0.65 & 0.02 \\
\hline PH46 & 0.18 & -0.62 & 0.12 & PH46 & -0.13 & -0.39 & 0.12 & PH53 & 0.26 & -0.91 & 0.02 \\
\hline \multirow[t]{2}{*}{$\begin{array}{l}\text { PH4 } \\
\text { PH17 }\end{array}$} & $\begin{array}{c}0.19 \\
0.1\end{array}$ & $\begin{array}{l}-0.29 \\
-0.21\end{array}$ & $\begin{array}{l}0.02 \\
0.18\end{array}$ & $\begin{array}{l}\text { PH4 } \\
\text { PH1 7 }\end{array}$ & $\begin{array}{l}-0.12 \\
-0.07\end{array}$ & $\begin{array}{l}-0.27 \\
-0.2\end{array}$ & $\begin{array}{l}0.24 \\
0.38\end{array}$ & & & & \\
\hline & & & & $\mathrm{PH} 2$ & -0.1 & -0.08 & 0.1 & \multirow[b]{2}{*}{ Item } & \multirow[b]{2}{*}{ Coef. } & \multirow[b]{2}{*}{ EE. } & \multirow[b]{2}{*}{ Sig. } \\
\hline Ittem & Coef. & E.E. & Sig. & Item & Coef. & E.E. & Sig. & & & & \\
\hline \multicolumn{4}{|c|}{ Libre de ansiedad (FRE) } & \multicolumn{4}{|c|}{ Popularidad (POP) } & \multicolumn{4}{|c|}{ Felicidad y satisfacción (HAP) } \\
\hline PH4 & 0.27 & - & - & $\mathrm{PH} 1$ & 0.54 & - & - & $\mathrm{PH} 2$ & 0.53 & - & - \\
\hline PH6 & 0.44 & -0.48 & 0 & $\mathrm{PH} 3$ & 0.45 & -0.14 & 0 & $\mathrm{PH} 8$ & 0.41 & -0.19 & 0 \\
\hline PH7 & 0.38 & -0.45 & 0 & PH6 & 0.04 & -0.14 & 0.47 & $\mathrm{PH} 28$ & 0.38 & -0.23 & 0 \\
\hline PH8 & 0.12 & -0.15 & 0.05 & PH1 1 & 0.03 & -0.12 & 0.31 & $\mathrm{PH} 31$ & 0.55 & -0.18 & 0 \\
\hline PH10 & 0.47 & -0.47 & 0 & PH32 & 0.42 & -0.17 & 0 & PH35 & 0.42 & -0.24 & 0 \\
\hline PH1 7 & 0.15 & -0.21 & 0.03 & $\mathrm{PH} 37$ & 0.36 & -0.14 & 0 & $\mathrm{PH} 40$ & 0.16 & -0.18 & 0 \\
\hline $\mathrm{PH} 23$ & 0.57 & -0.59 & 0 & PH39 & 0.05 & -0.15 & 0.58 & PH42 & 0.55 & -0.15 & 0 \\
\hline $\mathrm{PH} 29$ & 0.5 & -0.52 & 0 & PH41 & 0.42 & -0.15 & 0 & PH49 & 0.27 & -0.22 & 0 \\
\hline PH31 & -0.02 & -0.12 & 0.73 & PH47 & 0.55 & -0.13 & 0 & PH53 & 0.11 & -0.33 & 0.32 \\
\hline $\mathrm{PH} 32$ & 0.05 & -0.23 & 0.26 & PH51 & 0.18 & -0.12 & 0 & PH60 & 0.28 & -0.14 & 0 \\
\hline PH 35 & 0.21 & -0.26 & 0 & PH54 & -0.17 & -0.14 & 0 & PH2O & 0.17 & -0.19 & 0.01 \\
\hline PH4O & 0.1 & -0.2 & 0.08 & PH57 & 0.13 & -0.13 & 0.02 & $\mathrm{PH} 27$ & -0.2 & -0.21 & 0 \\
\hline PH56 & 0.56 & -0.59 & 0 & PH3O & -0.01 & -0.2 & 0.91 & PH3O & -0.37 & -0.53 & 0.02 \\
\hline \multirow[t]{7}{*}{ PH59 } & 0.41 & -0.41 & 0 & PH46 & 0.07 & -0.24 & 0.38 & PH16 & 0.28 & -0.22 & 0 \\
\hline & & & & PH53 & 0.2 & -0.18 & 0.12 & $\mathrm{PH} 25$ & -0.3 & -0.29 & 0 \\
\hline & & & & & & & & $\mathrm{PH} 43$ & 0.36 & -0.21 & 0 \\
\hline & & & & & & & & РН50 & 0.41 & -0.2 & 0 \\
\hline & & & & & & & & PH46 & -0.57 & -0.77 & 0.02 \\
\hline & & & & & & & & PH4 & 0.18 & -0.26 & 0.01 \\
\hline & & & & & & & & PH1 7 & 0.24 & -0.2 & 0 \\
\hline
\end{tabular}

Nota. Los coeficientes significativos son resaltados con letras cursivas y negrita. Los coeficientes de los reactivos representan las magnitudes de las relaciones estandarizadas de estos con la dimensión a la que pertenecen. Elaboración propia con los valores de la rutina lavaan del software $\mathrm{R}$ 
En la Tabla 3 se muestran las covarianzas entre los residuales que se encuentran en la misma dimensión, y que por ser reactivos cuyo contenido es muy similar, genera patrones identificables de respuestas entre los reactivos. Al establecer la covarianza entre los residuales mostrados, hubo mejora en los valores de ajuste del modelo. Es de resaltar que no se agotaron el número de covarianzas entre residuales.

Los resultados hasta aquí señalados sugieren que no existe unidimensionalidad en la prueba, por lo que en el análisis era prudente que durante la elaboración del modelo de medición se mantuviera la estructura de seis dimensiones en función al marco conceptual del instrumento. La Tabla 4 muestra las covarianzas entre las dimensiones de la escala de Autoconcepto, donde se observa que casi todas las dimensiones poseen entre sí una asociación positiva, excepto la dimensión de Comportamiento de Ajuste $(\mathrm{BEH})$ con Aspecto físico y atributos (PHY).
Los valores de ajuste mostrado por el modelo final obtenido fueron $\chi^{2}(1638)=2966.736$ $\mathrm{p}>0.05$; CFI (Comparative Fit Index) = 0.753; TLI (Tucker-Lewis Index) $=0.734$; , RMSEA (Root Mean Square Error of Approximation $)=0.041$; Intervalo de Confianza de RMSEA al 90\% $=0.038-0.043$. Por una parte, el modelo presenta valores de ajuste adecuados en $\chi^{2}$ y RMSEA, en tanto que los valores de ajuste de CFI y TLI no obtienen valores dentro del rango de lo aceptable. En consecuencia, la falta de buen ajuste del modelo propuesto puede ser atribuible a la falta de significatividad de varios coeficientes entre la relación de reactivos y las dimensiones a las que pertenecen, a saber, indicadores que pueden no estar representando el constructo con el que se le vincula; así como también porque el valor de los coeficientes es negativo cuando se espera una relación positiva entre reactivos y dimensiones. A lo señalado previamente debe añadirse la falta de una covarianza significativa entre las dimensiones Comportamiento de Ajuste (BEH) y Aspecto físico y atributos (PHY).

Tabla 3.

Resultados del análisis factorial confirmatorio AFC 3 en la versión Piers-Harris 2 aplicada en la muestra de niños mexicanos: covarianzas entre residuales de reactivos pertenecientes a la misma dimensión

\begin{tabular}{|c|c|c|c|c|}
\hline Dimensión & İtem & Coef. & E.E. & Sig. \\
\hline \multirow{2}{*}{ HAP } & $\mathrm{PH} 2 \leftrightarrow \mathrm{PH} 42$ & 0.39 & $(0.00)$ & 0.00 \\
\hline & $\mathrm{PH} 8 \leftrightarrow \mathrm{PH} 31$ & 0.23 & $(0.00)$ & 0.00 \\
\hline \multirow{3}{*}{ FRE } & $\mathrm{PH} 7 \leftrightarrow \mathrm{PH} 29$ & -0.18 & $(0.01)$ & 0.00 \\
\hline & PH7↔PH59 & -0.14 & $(0.01)$ & 0.01 \\
\hline & $\mathrm{PH} 32 \leftrightarrow \mathrm{PH} 1$ & -0.10 & $(0.01)$ & 0.08 \\
\hline \multirow[t]{2}{*}{ POP } & $\mathrm{PH} 1 \leftrightarrow \mathrm{PH} 37$ & -0.13 & $(0.01)$ & 0.01 \\
\hline & $\mathrm{PH} 41 \leftrightarrow \mathrm{PH} 1$ & -0.22 & $(0.01)$ & 0.00 \\
\hline \multirow{2}{*}{$\mathrm{BEH}$} & $\mathrm{PH} 12 \leftrightarrow \mathrm{PH} 19$ & 0.15 & $(0.00)$ & 0.00 \\
\hline & $\mathrm{PH} 12 \leftrightarrow \mathrm{PH} 27$ & -0.11 & $(0.00)$ & 0.03 \\
\hline
\end{tabular}

Nota. Los coeficientes significativos son resaltados con letras cursivas y negrita. 
Tabla 4.

Resultados del análisis factorial confirmatorio AFC 3 en la versión PiersHarris 2 aplicada en la muestra de niños mexicanos: covarianzas entre las dimensiones que conforman la escala.

\begin{tabular}{lccc}
\hline Dimensión & Coef. & E.E. & Sig. \\
\hline BEH & & & \\
INT & $\mathbf{0 . 6 3}$ & $(0.00)$ & 0.00 \\
PHY & 0.09 & $(0.00)$ & 0.27 \\
FRE & $\mathbf{0 . 2 7}$ & $(0.00)$ & 0.01 \\
POP & $\mathbf{0 . 5 3}$ & $(0.00)$ & 0.00 \\
HAP & $\mathbf{0 . 6 0}$ & $(0.00)$ & 0.00 \\
INT $\leftrightarrow$ & & & \\
PHY & $\mathbf{0 . 4 2}$ & $(0.00)$ & 0.00 \\
FRE & $\mathbf{0 . 5 2}$ & $(0.00)$ & 0.00 \\
POP & $\mathbf{0 . 4 9}$ & $(0.00)$ & 0.00 \\
HAP & $\mathbf{0 . 5 2}$ & $(0.00)$ & 0.00 \\
PHY $\leftrightarrow$ & & & \\
FRE & $\mathbf{0 . 3 2}$ & $(0.00)$ & 0.04 \\
POP & $\mathbf{0 . 3 4}$ & $(0.00)$ & 0.02 \\
HAP & $\mathbf{0 . 6 1}$ & $(0.00)$ & 0.01 \\
FRE $\leftrightarrow$ & & & \\
POP & $\mathbf{0 . 4 3}$ & $(0.00)$ & 0.00 \\
HAP & $\mathbf{0 . 1 9}$ & $(0.00)$ & 0.02 \\
POP $\leftrightarrow$ & & & \\
HAP & $\mathbf{0 . 6 5}$ & $(0.00)$ & 0.00 \\
\hline
\end{tabular}

Nota. Los coeficientes significativos son resaltados con letras cursivas y negrita.

El ajuste de los datos a un modelo Rasch de la escala de Autoconcepto de Piers-Harris 2, muestra que los reactivos de la escala tuvieron una bondad de ajuste infit con un rango de 0.88-1.32 (media -1.22); en tanto que en el ajuste outfit, la gran mayoría de los reactivos se encontró en un rango de 0.80-1.49 lógitos (promedio 0.99). Los ítems con valores fuera del rango previamente señalado fueron el 57 y 30, cuyos valores señalan la presencia de casos atípicos en ellos (1.70 y 2.37, respectivamente).

Con base en la Teoría de Respuesta al Ítem (IRT por sus siglas en inglés), se estimó la probabilidad de respuesta de los reactivos de la Escala de Autoconcepto Piers-Harris 2, cuyos resultados se muestran en la Figura 2. La distribución de ítems y respondientes se observan como dos histogramas verticales: los ítems al lado derecho identificando el número correspondiente del mismo en el instrumento o encuesta y se distribuyen desde aquellos que tienen la más alta, en la parte inferior, hasta los que menos probabilidad tienen de ser respondidos, en la parte superior. En dicha figura puede apreciarse que una gran cantidad de reactivos tienen una alta probabilidad de ser respondidos por tener valores por debajo de - 2 lógitos (15 de 60 reactivos); por otro lado, entre los valores de -2.0 a 0.0 lógitos, se encuentran la mitad de los reactivos que conforman la escala (33 de 60 ítems); finalmente, los ítems que tienen escasas probabilidades de ser respondidos se encuentran entre 0.0 y 1.0 lógitos ( 7 ítems). Solamente un reactivo tiene una muy baja probabilidad de probabilidad de respuesta, por encima de dos lógitos (ítem 30). 
Revista de Psicología y Ciencias del Comportamiento de la Unidad Académica de Ciencias Jurídicas y Sociales

Vol. 11. Núm. 2 (julio-diciembre 2020)

López-García, D.; Hernández-Padilla, E. y Palacios-Hernández, B.

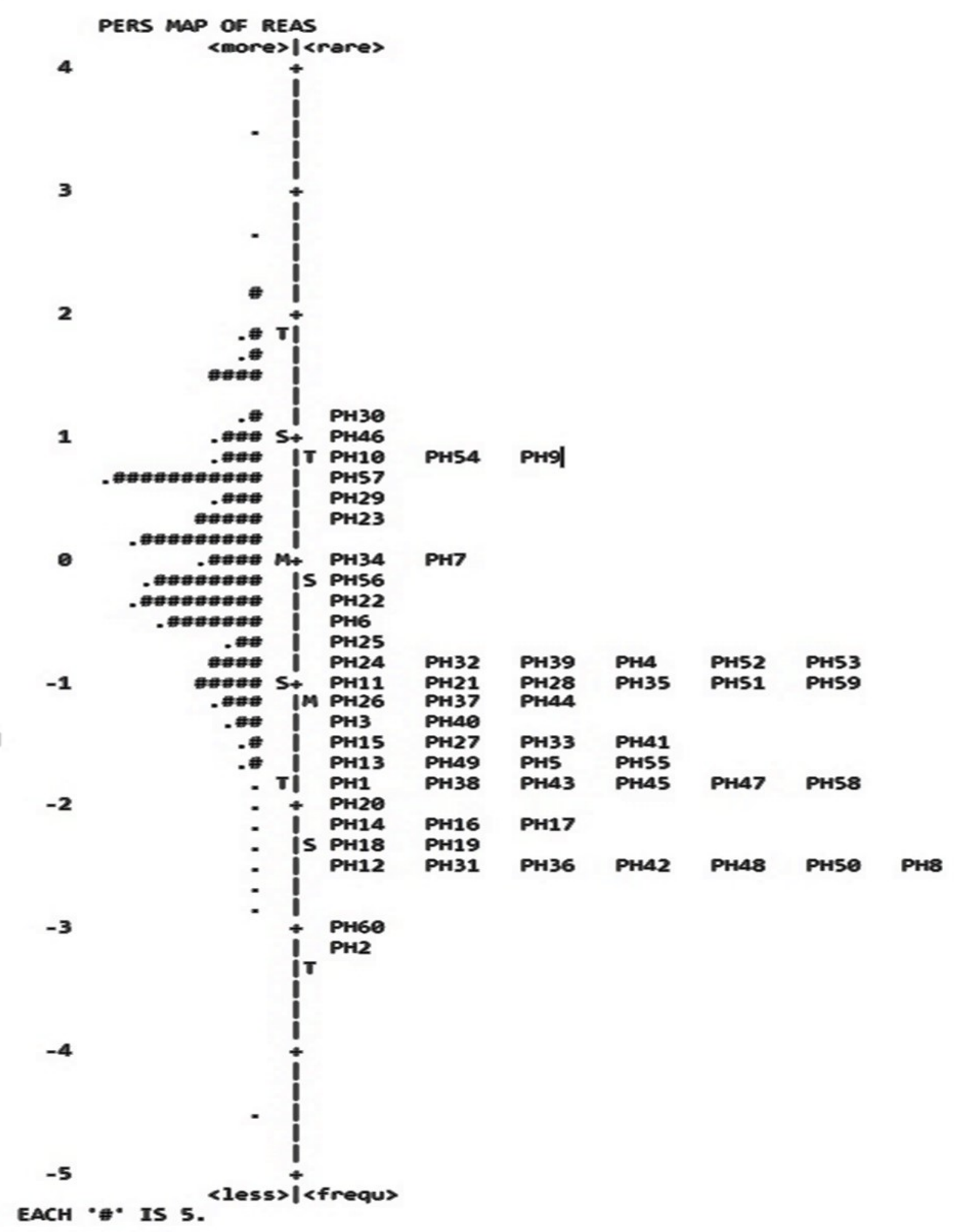

Figura 2. Mapa de Wright mediante el modelamiento Rasch de la Escala de Autoconcepto Piers-Harris 2. 


\section{Discusión}

Se aplicó la escala de Autoconcepto PiersHarris Segunda Edición en una muestra de 493 niños mexicanos en un rango de 7 a 12 años. Se obtuvieron los índices de confiabilidad aceptables mediante el procedimiento Kuder-Richardson 20 y con Alfa de Cronbach, que tuvieron la misma magnitud, encontrándose ligeramente por debajo de los reportados en la escala original (Piers \& Herzberg, 2002). Este resultado coincide con lo reportado en otros estudios (Rice, Kubal \& Preusser, 2004; Cardenal \& Fierro, 2003; Alexopoulos, \& Foudoulaki, 2002; Nishikawa, Norlander, Fransson, \& Sundbom, 2007), y en las diferentes dimensiones que lo componen (Flahive, Chuang \& Li, 2015; Butler \& Gasson, 2005). Cabe resaltar que la dimensión Popularidad obtuvo un coeficiente no aceptable, coincidiendo con otros estudios que reportan que esta dimensión muestra los índices más bajos de consistencia (George \& Mallery, 2003; Lemley, 2005; Streiner, 2003).

Las tendencias o similitudes entre los índices de confiabilidad de los estudios previamente citados deben ser consideradas con cautela debido a que la misma confiabilidad no es un valor inmutable, incluso al interior de la misma población; sujeta al tiempo, los cambios generacionales y los contextos socioculturales que deben siempre considerarse en estudios de adaptación y validación de instrumentos diseñados en otros contextos (Heine, 2001; Ramada-Rodilla, Serra-Pujadas \& Delclós-Clanchet, 2013). La confiabilidad puede ser mayor en tanto hay una mayor varianza en los puntajes totales, esto puede ser conseguido mediante una muestra más grande y heterogénea en la aplicación de cualquier escala (Streiner, 2003).

Los resultados del Análisis Factorial indicaron que de los cuatro índices de ajuste del modelo empírico solamente dos resultaron ser estadísticamente significativos. Esta consecuencia se podría atribuir a tres regularidades dentro del modelo empírico; la primera de ellas es la ausencia de coeficientes significativos entre indicadores o reactivos y las dimensiones a las que se dicen representar. Esto es coherente con lo reportado en otros trabajos empleando el análisis factorial exploratorio para la Escala de Autoconcepto Piers Harris 2, donde el número de factores que se reporta es variable_(Flahive, Chuang, \& Li, 2011; Butler \& Gasson, 2005; Lewis \& Knight, 2000), y, en consecuencia, los reactivos pueden representar dimensiones distintas a aquellas para las que fueron creados. La segunda regularidad refiere la presencia de coeficientes negativos, estadísticamente significativos, de los ítems con la dimensión a la que representan; este resultado podría sugerir que el reactivo mide en sentido opuesto el atributo, o bien, la existencia de una relación negativa entre ellos que afecta la bondad de ajuste del modelo. Finalmente, en tercera posición puede observarse (Tabla 4) la falta de una covarianza significativa entre las dimensiones Comportamiento de Ajuste (BEH) con Aspecto físico y atributos (PHY), la cual afecta la representación de la estructura de las dimensiones de la escala, puesto que no todas se interrelacionan, afectando el ajuste del modelo empírico.

Por su parte, el modelamiento Rasch de la escala reportó que los reactivos que la integran poseen buena bondad de ajuste tanto para las respuestas dentro del límite de medida del ítem, así como de los valores extremos de éste. Asimismo, una peculiaridad de la escala es la baja probabilidad de respuesta en la mayoría sus ítems. Gracias al uso del modelo de Rasch se identificó que en el instrumento: a) la apreciación de los jueces expertos y docen- 
tes, deben considerar muy importante la interpretación del significado de palabras y sentencias que los participantes lean; muestra de ello es la falta de ajuste de dos ítems a los valores extremos (PH2 y $\mathrm{PH} 60)$; de este punto surge la recomendación de incluir durante el proceso de validación entrevistas previas con participantes representativos de la población objetivo de la prueba para realizar un sondeo pertinente que identifique su nivel de competencia en el tema a evaluar. Por otra parte, b) mediante el mapa de Wright de la escala, se observó que ésta adolecía de ítems con bajas probabilidades de respuesta, lo que impide discriminar a los respondientes con valores altos y muy altos del autoconcepto. Ambas problemáticas podrían corregirse en futuras aplicaciones incluyendo ítems de baja probabilidad de respuesta, o bien la adaptación de los existentes para que discriminen a los respondientes con altas probabilidades de responder esos reactivos, mediante una valoración más amplia por parte de jueces expertos $\mathrm{y}$ docentes.

\section{Conclusiones}

En el presente estudio puede observarse que los respondientes de la Escala de Autoconcepto Piers Harris 2 muestran consistencia en la forma que manifiestan sus opciones de respuesta en todas las dimensiones, excepto en una (Popularidad) en la que debe haber un mayor énfasis o cuidado. A través del AFC puede apreciarse una falta de ajuste de modelo de manera marginal que puede ser mejorada en una revisión del contenido de los reactivos; no obstante, esta falta de conformación de dimensiones claramente identificables es algo recurrente en los diversos estudios y adaptaciones que se han hecho de la prueba, pese a ello los resultados de la escala deben ser interpretados con cautela y/o con una vali- dez concurrente. Respecto a la probabilidad de respuesta a los reactivos, mediante análisis IRT se requieren reactivos con menor probabilidad de respuesta para discriminar al sector de la población que muestre valores de alto o muy alto autoconcepto en sus distintas dimensiones. Finalmente, se considera que el uso de la escala en muestras de población mexicana con semejante rangos de edad y escolaridad puede realizarse ya que se reportaron los índices de confiabilidad y consistencia interna adecuados.

\section{Agradecimientos}

Al Consejo Nacional de Ciencia y Tecnología (CONACyT) de México por la beca doctoral asignada a la primera autora que permitió la realización del estudio.

\section{Referencias}

Alrajhi, M., Aldhafri, S., Alkharusi, H., Alharthy, I., Albarashdi, H. \& Alhadabi, A. (2019). Grade and Gender Effects on Self-Concept Development. The Open Psychology Journal, 12, 66- 75. doi:10.1093/geronb/56.4.P195

Alexopoulos, D. S. \& Foudoulaki, E. (2002). Construct Validity of the Piers-Harris Children's SelfConcept Scale. Psychological Reports, 91(3), 827-838 doi:10.2466/pr0.2002.91.3.827

Becker, G. (2008). On the integrity of reliability estimation in classical test theory: the case for an additive coefficient of stability. Psychological Reports, 103, 545-565. doi:10.2466/ pr0.103.2.545-565

Butler, R. J. \& Gasson, S. L. (2005). Self Esteem/Self Concept Scales for Children and Adolescents: A Review. Child and Adolescent Mental Health, 10(4), 190-201. doi:10.1111/j.14753588.2005.00368.x

Cardenal, V. y Fierro, A. (2003). Componentes y cor relatos del autoconcepto en la escala de PiersHarris. Estudios de Psicología: Studies in Psy chology, 24(1), 101-111. doi:10.1174/021093903321329094

Cazalla, M. N. y Molero, D. (2013). Revisión teórica sobre el autoconcepto y su importancia en la adolescencia. Revista Electrónica de Investigación y Docencia (REID),10, 43-64. Recupe- 
rado de: https://revistaselectronicas.ujaen.es/ index.php/reid/article/view/991

Collins, L. C., Kafer, N. F. \& Shea, J. D. (1985). The Piers-Harris Children's self-concept scale: An Australian study. Australian Psychologist, 20 (2), 177-193. doi:10.1080/00050068508256164

Cvencek, D., Fryberg, S. A., Covarrubias, R. \& Metzoff, A. N. (2018). Self-Concepts, SelfEsteem, and Academic Achievement of Minority and Majority North American Elementary School Children. Child Dev, 89(4), 10991109. doi:10.1111/cdev.12802

Chavez-Hernandez, A. M., Correa-Romero, F. E., Acosta-Rojas, I. B. Cardoso-Espindola, M. K. V., Padilla-Gallegos, G. M. \& ValadezFigueroa, I. (2018). Suicidal Ideation, Depressive Symptomatology, and Self-Concept: A Comparison Between Mexican Institutionalized and Noninstitutionalized Children. Suicide and Life-Threatening Behavior, 48(2), 193-198. doi:10.1111/sltb. 12340

Dolgun, D. Savaşer, S. \& Yazgan, Y. (2014). Determining the correlation between quality of life and self-concept in children with attention deficit/hyperactivity disorder. Journal of Psychiatric and Mental Health Nursing, 21, 601608. doi:10.1111/jpm.12114

Ekinci, O., Isik, U., Gunes, S., Yildirim, C., Killi, C. \& Gülen, G. (2016). Self-concept in children and adolescents with epilepsy: The role of family functioning, mothers' emotional symptoms and ADHD. Brain \& Development, 38(8), 714-722. doi:10.1016/j.braindev.2016.02.015

Fives, A. (2016). The association of attitude to reading and reading achievement among a representative sample of nine-year olds in Ireland. Reading Psychology, 37, 27-54. doi:10.1080/02702711.2014.977983

Flahive, M. W., Chuang, Y. -C. \& Li, C.-M. (2011). Reliability and Validity Evidence of the Chinese Piers-Harris Children's Self-Concept Scale Scores Among Taiwanese Children. Journal of Psychoeducational Assessment, 29 (3), 273-285. doi:10.1177/0734282910380191

Flahive M-hW, Chuang, Y-C. \& Li C-M. (2015). The Multimedia Piers-Harris Children's SelfConcept Scale2: Its Psychometric Properties, Equivalence with the Paper-and-Pencil Version, and Respondent Preferences. PLoSONE, 10(8), 1-13. doi:10.1371/ journal.pone.0135386
García, F. y Musitu, G. (1999). AF-5. Autoconcepto Forma 5. Madrid, España: TEA Ediciones.

Goltz, H. \& Brown, T. (2014). Are children's psychological self-concepts predictive of their selfreported activity preferences and leisure participation. Australian Occupational Therapy Journal, 61, 177-186. doi:10.1111/14401630.12101

Galindo-Domínguez, H. (2019). Estandarización por Curso y Género de la Escala de Autoconcepto

AF-5 en Educación Primaria. Psicología Educativa, 25(2), 117-125. doi:10.5093/ psed2019a9

George, D. \& Mallery, P. (2003). SPSS for Windows step by step: A simple guide and reference.

11.0. Update. Boston: Allyn \& Bacon.

Gorostegui, M. E. y C. Rogers. (Ed.). (1992). Adaptación y construcción de normas para Chile de la Escala de Autoconcepto para niños de Piers-Harris. Libertad y creatividad en la educación. El sistema no directivo. Buenos Aires, Argentina: Paidós

Gorostegui, M. E. (2010). Adaptación de un instrumento de evaluación de autoestima para adolescentes: el desafío para la psicometría. [Tesis de Doctorado en Educación]. Universidad Academia de Humanismo Cristiano. Chile. Recuperado de: http:// bibliotecadigital.academia.cl handle/123456789/684

Guay, F., Marsh, H. W. \& Boivin, M. (2003). Academic self-concept and academic achievement: Developmental perspectives on their causal ordering. Journal of Educational Psychology, 95(1), 124-136. doi:10.1037/00220663.95.1.124

Guido, G. P., Mújica S. A. y Gutiérrez, M. R. (2011). Diferencias en el autoconcepto por sexo en la adolescencia: construcción y validación de un instrumento. LIBERABIT, 17(2): 139-146. Recuperado de: https:/www.redalyc.org/ articulo.oa? id $=686 / 68622584004$

Ha, P., Shi, B., y \& Zheng, Q. (2015). Self-concept of Chinese children and adolescents with cleft lip and/or palate. International Journal of Oral and Maxillofacial Surgery, 44, 219-220. doi:10.1016/j.ijom.2015.08.111

Heine, S. J. (2001). Self as cultural product: An examination of East Asian and North American selves. Journal of Personality, 69, 881-906. doi:10.1111/1467-6494.696168 
Kuder, G. F. \& Richardson, M. V. (1937). The theory of the estimation of test reliability. Psychometrika, 2(3), 151-60. doi:10.1007/ BF02288391

Lemley, N. N. (2005). The Reliability of the PiersHarris Children's Self-Concept Scale, Second Edition. [Tesis del Graduate College of Marshall University]. South Charleston. Estados Unidos de Norteamérica.

Lewis, J. D. \& Knight, H. V. (2000). Self-Concept in Gifted Youth: An Investigation Employing the Piers-Harris Subscales. Gifted Children Quarterly, 14(1), 45-53. doi:10.1177/001698620004400105

Nishikawa, S., Norlander, T., Fransson, P., \& Sundbom, E. (2007). A cross-cultural validation of adolescent self-concept in two cultures: Japan and Sweden. Social Behavior and Personality, 35, 269-286. doi:10.2224/ sbp.2007.35.2.269

Pérez-Gil, J., Chacon, S. y Moreno, R. (2000). Validez de constructo: el uso del análisis factorial exploratorio-confirmatorio para obtener evidencias de validez. Psicothema, 12 (2) 442446. Recuperado de: https://www.redalyc.org/ articulo.oa?id $=727 / 72797102$

Piers, E. V. (1984). Piers-Harris Children's SelfConcept Scale. Revised Manual. Los Angeles: Western Psychological Services.

Piers, E. \& Herzberg, D. (2002). Piers-Harris Children's Self Concept Scale-Second Edition Manual. Los Ángeles, CA: Western Psychological Services.

Pineda, S. E. y Pérez, R. M. (2015). Autoconcepto en adolescentes y adultos: construcción, validez y confiabilidad de un instrumento. Por una Psicología sin fronteras, 249-269. Recuperado de: https:www.academia.edu/21796666/ Auco-

ncto_en_adolescentes_y_adultos_Construcci $\% \mathrm{C} 3 \%$

B3n_validez_y_confiabilidad_de_un_instrum ento

Ramada-Rodilla, J.M., Serra-Pujadas, C. y DelclósClanchet, G.L. (2013). Adaptación cultural y validación de cuestionarios de salud: revisión y recomendaciones metodológicas. Salud Pública Mex; 55, 57-66. Recuperado de: http:// www.scielo.org.mx/pdf/spm/v55n1/ v55n1a09.pdf

R Development Core Team. (2018). $R$ : A language and environment for statistical computing [Software de computación]. Recuperado de: http://www.R-project.org
Rice, K. G.; Kubal, A. E. \& Preusser. K. (2004). Perfectionism and children's self-concept: further validation of the adaptive/maladaptive perfectionism scale. Psychology in the Schools, 41(3), 279-290. doi:10.1002/ pits. 10160

Salum-Fares, A., Marín, R. y Reyes, C. (2011). Relevancia de las dimensiones del autoconcepto en estudiantes de escuelas secundarias de Ciudad Victoria, Tamaulipas, México. Revista Electrónica de Psicología Iztacala, 14(2), 255272. Recuperado de: https:// www.medigraphic.com/pdfs/epsicologia/epi2011/epi112n.pdf

Shavelson, R., Hubner, J. \& Stanton, J. (1976). Selfconcept: Validation of construct interpretation. Review of Educational Research, 46(3), 407-441. doi:10.3102/00346543046003407

Shields, N., Taylor, N.F. \& Dodd, K.J. (2008). Selfconcept in children with spina bifida compared with typically developing children. Develop mental Medicine \& Child Neurology, 50, 733743. doi:10.3102/00346543046003407

Streiner, D. L. (2003). Starting at the beginning: an introduction to coefficient Alpha and inter nal consistency, Journal of Personality Assess ment, 80(1), 99-103. doi:10.1207/ S15327752JPA8001_18

Susperreguy, M.I., Davis-Kean, P.E., Duckworth, K. \& Chen, M. (2018). Self-Concept Predicts Academic Achievement Across Levels of the Achievement Distribution: Domain Specificity for Math and Reading. Child Development, 89(6), 2196-2214. doi:10.1111/ cdev. 12924

Zimmerman, D. W. (1972). Test reliability and the Kuder-Richardson formulas: derivation from probability theory. Educational and Psychological Measurement, 32, 939-954. doi:10.1177/001316447203200408 
